International Journal of Computer Networks \& Communications (IJCNC) Vol.4, No.3, May 2012

\title{
FUZZY-LOGIC ADAPTIVE QUEUING FOR A Heuristic TCP PERFormanCE IN MOBILE WIRELESS NETWORKS
}

\author{
Ghaida A. AL-Suhail, Turki Y. Abdallah, and Omar Majid ${ }^{1}$ \\ ${ }^{1}$ Department of Computer Engineering, University of Basrah, Basrah, IRAQ \\ ghaida_alsuhail@yahoo.com, protryounis@yahoo.com \\ omkh88@yahoo.com
}

\begin{abstract}
In this paper, we propose a new Fuzzy-Logic Adaptive Queuing controller (FLAQ) based on a classical Random Early Detection (RED) algorithm in wireless cellular network. The controller predicts dynamically the packet dropping rate and the corresponding average queue length. It relies on the average queue length at the base station router and the packet loss rate caused by the channel variations in mobile environment; assuming there is no buffer overflow due to the congestion. Using this model, a heuristic TCP performance can be estimated over a time-varying channel under different conditions of user's mobility. The results show a significant improvement in TCP throughput performance when the user's mobility is below $5 \mathrm{~m} / \mathrm{s}$; and becomes constant (i.e., close to i.i.d) beyond this speed especially at $5 \%$ of predefined packet error rate.
\end{abstract}

\section{KEYWORDS}

Fuzzy Logic, Queue Management, TCP Throughput, Time-Varying Channel, Wireless \& Mobile Network

\section{INTRODUCTION}

Computer networks like internet necessitate network resources (i.e. bandwidth, buffer spaces, etc.) in order to accommodate the arriving packets at router buffers [1][2]. When the arriving packets cannot be accommodated due to lack of network resources, this indicates occurring congestion at router buffers of networks. More specifically, a poor network performance due to congestion can be offered in terms of high dropping and queuing delay for packets, low throughput and not maintaining the average queue length which may not prevent the router buffers from building up, then dropping packets. Moreover, congestion may also trigger an unbalanced share among the network sources [3].

However, congestion control mechanism implemented through "Queue Management" algorithms, is still the key factor to solve this problem keeping TCP/IP networks efficient and reliable from the user's viewpoint. In particular, it is well-known that the rapidly emerging mobile data networks such as 3G, HSPA, and LTE and the integrated WAN, LAN and 3G cellular systems has created new and crucial challenges for the development of Internet applications [4][5]. Unlike the wired networks, the mobile networks exhibit some challenges basically related to two factors; $(i)$ a highly variable bandwidth and (ii) base stations are often equipped with large buffers in order to absorb bandwidth fluctuations and consequently prevent the unnecessary packet loss. Therefore, TCP/IP needs to meet the challenges introduced by the wireless portion of the network in order to make it valid with the wired network protocols for providing efficient services by web accesses, e-mails, bulk data transfers, etc. To solve and 
International Journal of Computer Networks \& Communications (IJCNC) Vol.4, No.3, May 2012

mitigate these challenges, many works have investigated methods to estimate the key wireless link parameters, for example, link bandwidth estimation [6], link buffer size estimation such as max-min, loss-pair and sum-of delays [7]-[9], and queue length estimation [10]. For this reason, the utilization of fuzzy logic (FL) has shown to be useful in designing new active queue management (AQM) methods [11]-[16] that can be used to alleviate congestions in wired and wireless networks. For example, Mallapur et al. [15] propose buffer manager located at the base station using a fuzzy controller for packet dropping in wireless cellular networks. The controller uses three fuzzy parameters, namely application priority, queue length and packet size.

The traditional technique for managing queue lengths is to set a threshold (in terms of packets) for each queue, accepts packets for the queue until the threshold is reached, then reject (drop) subsequent incoming packets until the queue decreases below the value of threshold. This technique is known as "tail drop". This threshold most of the time is the maximum capacity of buffer, then tail drop signals congestion only when the queue has become full. The solution to the full queues problem is for routers to drop packets before a queue becomes full, so that end nodes can respond to congestion before buffers overflow. By dropping packets before buffers overflow, AQM allows routers to control packet drops. Many researches have been devoted on the queue size management algorithms such as Random Early Detection (RED) [16] and Weighted RED [17], PID controller [18], and NLRED [3] to reduce the likelihood of global synchronization, as well as keeping queue sizes down in the face of heavy load and bursty traffic. Other related AQM schemes such as GRED, WRED, and ARED are summarized in [3]. The Random Early Detection (RED) mechanism can solve the Drop Tail's (DT) deficiencies well. It uses randomization to ensure that all connections encounter the same loss rate. By dropping packets before the router's buffers are completely exhausted, the RED mechanism try to prevent congestion. In [19], another approach known as Random Exponential Marking (REM) was also developed to measure the average queue size instead of congestion measure. It was able to achieve high link utilization, negligible packet loss and short queuing delay in a simple scalar manner.

Modeling the dynamics of TCP and AQM is another important research area in heterogeneous networks. Accordingly, it is found that the main degradation of Quality of Service (QoS) in the wired networks is the congestion due to buffer overflow at the routers. Meanwhile, the QoS degradation in wireless links occurs not only caused by the congestion, but also due to possible high bit errors of the radio channels. Although TCP is basically employed for end-to-end wired Internet, it can not achieve sufficient throughput in the wireless environment where packet are lost due to transmission errors. TCP assumes that losses of packets are derived from a buffer overflow at a BS router. It invokes the congestion control to reduce traffic unnecessarily when the packet loss occurs due to transmission errors on a wireless link. Thus one approach to mitigate this problem and distinguish packet loss due to congestion and others packet errors due to channel variations, is to employ TCP proxy called Performance Enhancing Proxy (PEP) [20].

In particular, the approaches devoted on improving TCP throughput over wireless cellular networks can be classified as follows. (i) Splitting connection at the wired-wireless border (e.g., BS). However, the major problem of such Indirect-TCP (e.g. Snoop protocol) and M-TCP is a hand-off latency due to mobility of MH [5][21]. Explicit loss notification (ELN) is another more precise approach with capability of distinguishing the packet loss due to packet loss due to congestion and the one from transmission errors. (ii) Developing data-link layer protocols, e.g. Automatic Repeat request (ARQ) and/or adaptive FEC [21] and (iii) Designing Cross-layer approaches [22] where some approaches focuses on RED AQM over wireless channels [23][24]. The channel variations are mainly caused due to the highly or correlated bit errors and this in consequence can cause to packet dropping and eventually reduce the network throughput. In this case, a TCP proxy at the router queue of the base station can be employed to distinguish 
International Journal of Computer Networks \& Communications (IJCNC) Vol.4, No.3, May 2012

the packet error (dropping) due to the variations of wireless channel and the packet loss due to congestion of buffer overflow.

In this paper, therefore we develop a new Fuzzy-Logic Adaptive Queuing controller (FLAQ)RED at the base station in order to tune the average queue length and the wireless packet error. The proposed model inclines to maintain small buffer size space over a time-varying channel which may exhibit significant degradation in the network bandwidth estimation. Then, we predict the lowest packet dropping received by the mobile receiver over Rayleigh fading environment when there is no congestion effect at the Base station buffer. By using this model, a heuristic TCP performance can be evaluated over a time-varying channel under different conditions of user's mobility.

\section{NetWork Preliminaries}

\subsection{Classical RED}

The RED scheme operates as follows. RED computes the average queue size $Q_{\text {ave }}$ and a drop probability $P_{d}$ based on the instantaneous queue size. In addition, RED maintains two thresholds of queue size $Q_{\min }$ and $Q_{\max }$. Figure 1 explains the basic operation of RED as follows [17]:

If $Q_{\text {avg }}<Q_{\min }$, no packet drop

If $Q_{\min } \leq Q_{\text {avg }} \leq Q_{\max }$, drops each packet with the probability $\mathrm{P}_{\mathrm{d}}$.

If $Q_{\text {avg }}>Q_{\max }$, drop every arriving packet.

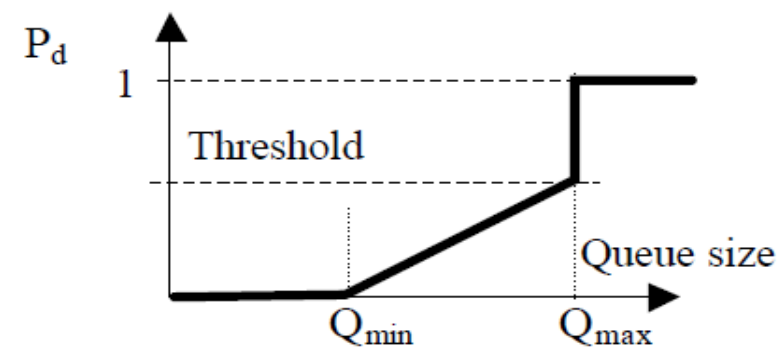

Figure 1. A Classical RED Algorithm

In the RED mechanism, the average queue size, $Q_{\text {ave }}$, is calculated and compared with two thresholds, a minimum threshold $\left(\min _{\mathrm{th}}\right)$ and a maximum threshold $\left(\max _{\mathrm{th}}\right)$. When $Q_{\text {avg }}$ is less than the minth, no packets are marked. When it is greater than the $\mathrm{Q}_{\max }$ every arriving packet is marked.

When $Q_{\text {ave }}$ is between the minth and the maxth, each arriving packet is dropped with probability $P_{a}$, where $P_{a}$ is a function of $Q_{a v e}$. The packet-marking probability $P_{b}$ is calculated as below [17]:

$$
P_{b}=\frac{\left.\max Q_{a v g}-\max t h\right)}{(\max t h-\min t h)}
$$

The final packet-dropping probability $\mathrm{P}_{\mathrm{a}}$. increases slowly as the count increases since the last marked packet: 
International Journal of Computer Networks \& Communications (IJCNC) Vol.4, No.3, May 2012

$$
P_{a}=\frac{P_{b}}{1-\operatorname{count} \times P_{b}}
$$

\subsection{Network Model}

We consider a common scenario of unicast network topology model of a wired-to-wireless network to investigate the TCP performance over wireless link as shown in Figure 2. The network model consists of the fixed host $(\mathrm{FH})$ which is defined over the wired link, while the wireless last hop consists of a single pair of base station (BS) and one mobile host (MH) in wireless cellular network [21][24]. Within this model, the wireless channel feedback signal in terms of TCP ACK is used to return back the estimation of channel variations in terms of the wireless packet error rate (PER) over wireless channel.

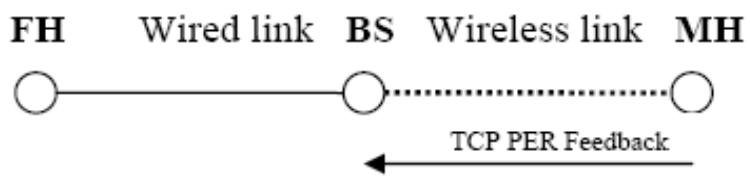

(a)

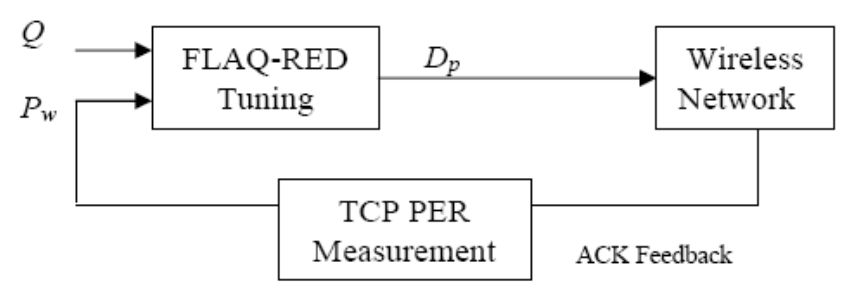

(b)

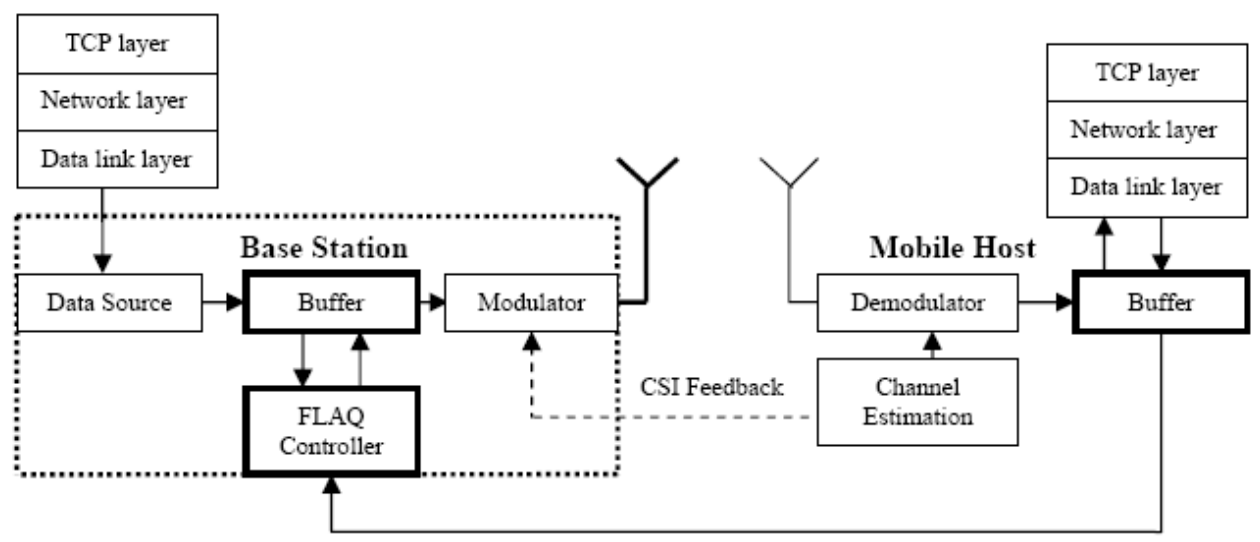

Round-Trip Time (ACK) - Channel feedback

(c)

Figure 2.The proposed approach $\quad$ (a) Unicast Network Model $\quad$ (b) The process of proposed FLAQ-RED and (c) System model based on FLAQ-RED

\subsection{System Scenario}

We consider the key preliminaries required to predict the packet dropping rate based on fuzzy logic adaptive queuing scheme over Rayleigh fading channel as follows: 
International Journal of Computer Networks \& Communications (IJCNC) Vol.4, No.3, May 2012

- A single TCP flow traffic is considered for only one mobile receiver.

- The network is assumed to be stable without heavy or bursty TCP traffic.

- The TCP packet error rate (PER), (i.e., $P_{w}$ ), is caused by the variations of wireless channel when only highly bit errors occurs during traffic transmission. Assuming there is no congestion at the router buffer of BS.

- $\quad P_{w}$ is measured by the channel estimator at mobile receiver and returned back via the ACK feedback of the round trip of TCP to indicate the sender about the channel bit errors, so we consider $P_{w}$ changes from 5\% to $30 \%$.

- The TCP proxy at the router queue of the base station is required if and only if multiple TCP flows are present [20][21][24]. This proxy is mainly used to distinguish the packet error (dropping) due to the variations of wireless channel and the packet loss due to congestion of buffer overflow. In our system scenario, there is no packet dropping due to any buffer overflow. So, the link is under- utilization bandwidth.

- At the base station (BS), we consider the following assumptions:

- $\quad$ Let the buffer size $=200$ packets

- $\quad$ The router queue with $Q_{\min }=50$ [packet] , and $Q_{\max }=150$ [packet]

- If the average queue length less than 50 no packets are dropped

- If the average queue length more than 150 all the arriving packets are dropped

- If the average queue length between $Q_{\min }$ and $Q_{\max }$ then the dropped packets controlled by the fuzzy logic controller depending on to inputs ( $A V Q L$ and $P_{w}$ )

- Packet loss rate at base station is ignored (no packet loss due to congestion)

\section{Proposed FLAQ-RED CONTROLler}

Fuzzy logic controllers FLC, like expert systems, can be used to model human experiences and human decision-making behaviours [25]. In FLC the input-output relationship is expressed by using a set of linguistic rules for relational expressions. An FLC basically consists of four conceptual components including a fuzzifier, a defuzzifier, an inference engine and a rule base.

Figure 3 shows the Fuzzy Logic Controller architecture. It is evident that as in many fuzzy control applications, the input data are usually crisp, so a fuzzification is necessary to convert the input crisp data into a suitable set of linguistic value that is needed in inference engine. Singleton fuzzifier maps the crisp input to a singleton fuzzy set.

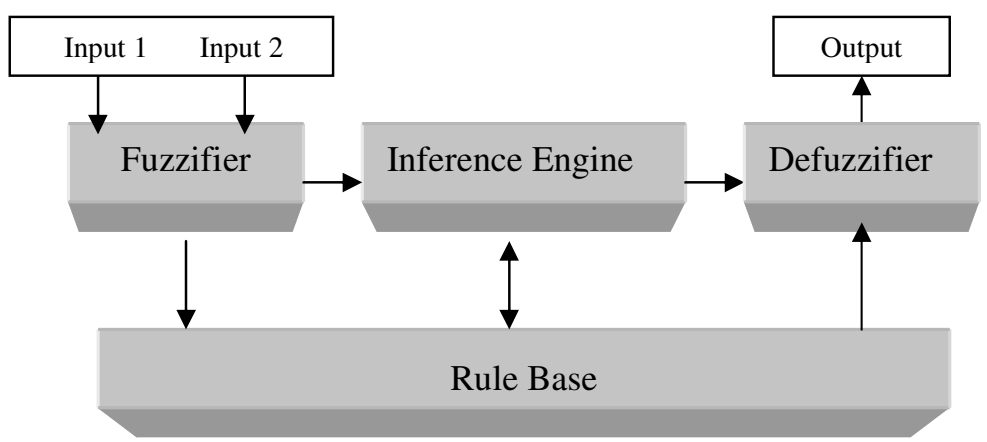

Figure 3.Fuzzy Logic Controller Architecture 
International Journal of Computer Networks \& Communications (IJCNC) Vol.4, No.3, May 2012

In the rule-base of an FLC, a set of fuzzy control rules, which characterize the dynamic behaviour of system, are defined. The inference engine is used to form inferences and draw conclusions from the fuzzy control rules. The output of inference engine is sent to defuzzification unit. Defuzzification is a mapping from a space of fuzzy control actions into a space of crisp control actions.

To implement FLAQ-RED scheme, we use a combination of two methods [11] to construct the fuzzy linguistic rules: (1) The trail and error and (2) The theory method. This is because the first method depends on domain expert knowledge and experience, and the second method tunes the input and output linguistic parameters to accurate values. Thus using both methods together, the output $D_{\mathrm{p}}$ can be obtained in more accurate.

In proposed FLAQ-RED, FLC consists of (1) fuzzification (2) evaluation of the rules, (3) aggregation the outputted rules, and (4) defuzzification, to compute the output $D_{p}$ at the BS router queue. Then, the FLAQ employs two input linguistic variables $\left(A V Q L, P_{w}\right)$ with aim to evaluate a single output linguistic variable $\left(D_{\mathrm{p}}\right)$. The general bell membership function is used to represent all linguistic variables. The generalized bell function depends on three parameters $a, b$, and $c$ as given by

$$
f(x ; a, b, c)=\frac{1}{1+\left|\frac{x-c}{a}\right|^{2 b}}
$$

The parameter $b$ is usually positive and the parameter c locates the centre of the curve. Then, every linguistic variable is linked to a fuzzy set of the input and output linguistic variables as defined in Table 1:

Table (1): Linguistic variables ranges

\begin{tabular}{ll}
\hline \multicolumn{1}{c}{ Variables ranges } & Linguistic variables \\
\hline$A V Q L=50-150$ [packet $]$ & $A V Q L=\{$ low, medium, high, very high $\}$. \\
$P_{w}=5 \%-30 \%$ & $P w=\{$ low, medium, high, very high $\}$. \\
$D_{\text {predict }}=0 \%-30 \%$ & D_predicted $=\{$ very low, low , medium, high, very high $\}$. \\
\hline
\end{tabular}

\section{Fuzzy Rules Base:}

Fuzzy rules are linguistic IF-THEN constructions that have the general form "IF $A$ THEN $B$ " where $A$ and $B$ are propositions containing linguistic variables. A is called the premise and $\mathrm{B}$ is the consequence of the rule. The type of any fuzzy system is specified by the type of the rules used in it. Generally there are two types of fuzzy systems known as Mamdani and TakagiSugeno-Kang (TSK) fuzzy systems [26].

An example of fuzzy rule for $m$ inputs $(x 1, x 2, \ldots, x m)$ and only one output y (Multi Input Single Output (MISO)) could be defined in many forms like:

Mamdani Type: $R$ : If $x 1$ is $\mu(x 1)$ and $x 2$ is $\mu(x 2)$ and ... and $x m$ is $\mu(x m)$ then $y$ is $\mu(y) \ldots$ Or

Sugeno Type: $\quad$ : If $x 1$ is $\mu(x 1)$ and $x 2$ is $\mu(x 2)$ and ... and $x m$ is $\mu(x m)$ then $y=f(x 1, . ., x m) \ldots$ 
International Journal of Computer Networks \& Communications (IJCNC) Vol.4, No.3, May 2012

Because $A V Q L$ has four membership functions and also $P_{w}$ has four membership functions, then there are 16 fuzzy rules can be generated as listed in Table 2. As a result, Figure $\mathbf{4}$ depicts the details of the proposed Fuzzy-Logic Adaptive Queuing based RED scheme using Mamdani Fuzzy -Logic when General Bell function of (3) is considered. The memberships of linguistic variables of $A V Q L, P_{w}$ and $D_{p}$ are adjusted depending on the wireless environment in order to introduce the resultant fuzzy logic system decision surface.

Table (2): Fuzzy Rule-Base System

Fuzzy Rule Base System of FLAQ based RED

If (AVQL is low) and (pw is low) then (D_predicted is very__low)

If (AVQL is low) and (pw is medium) then (D__predicted is very_low)

(AVQL is low) and (pw is high) then (D_predicted is very_low)

(AVQL is low) and (pw is very_high) then (D_predicted is low)

(AVQL is medium) and (pw is low) then (D_predicted is very_low)

(AVQL is medium) and (pw is medium) then (

(AVQL is medium) and (pw is high) then (D_predicted is low)

(AVQL is medium) and (pw is very__high) then (D_predicted is medium)

(AVQL is high) and (pw is low) then (D_predicted is very_low)

(AVQL is high) and (pw is medium) then (D_predicted is low)

(AVQL is high) and (pw is high) then (D_predicted is medium)

(AVQL is high) and (pw is very__high) then (D__predicted is high)

(AVQL is very_high) and (pw is low) then (D_predicted is low)

(AVQL is very_high) and (pw is medium) then (D__predicted is medium)

(AVQL is very_high) and (pw is high) then (D_predicted is high)

(AVQL is very__high) and (pw is very_high) then (D_predicted is very_high)

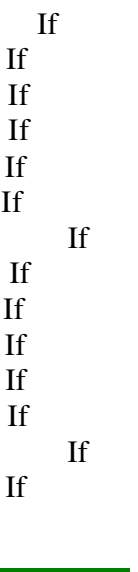

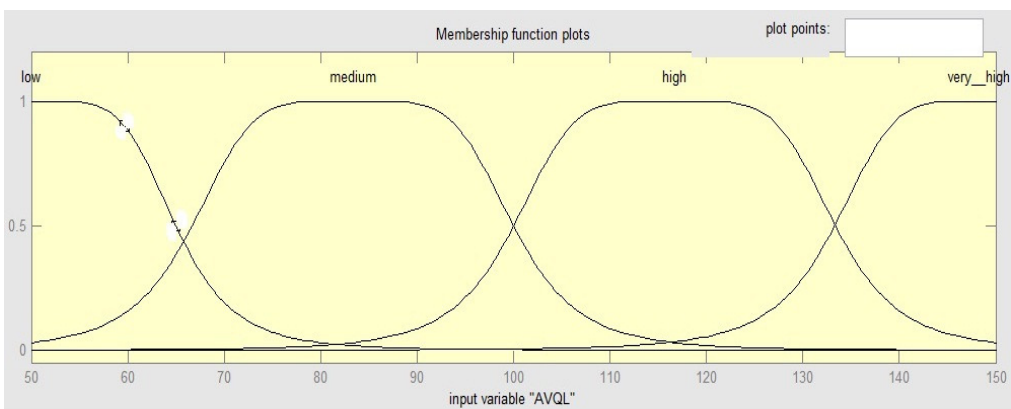

(a)

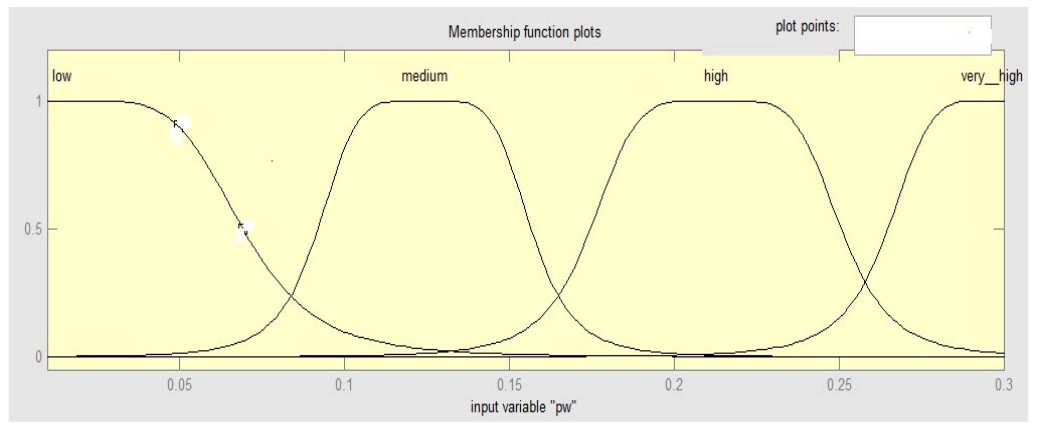

(b) 
International Journal of Computer Networks \& Communications (IJCNC) Vol.4, No.3, May 2012

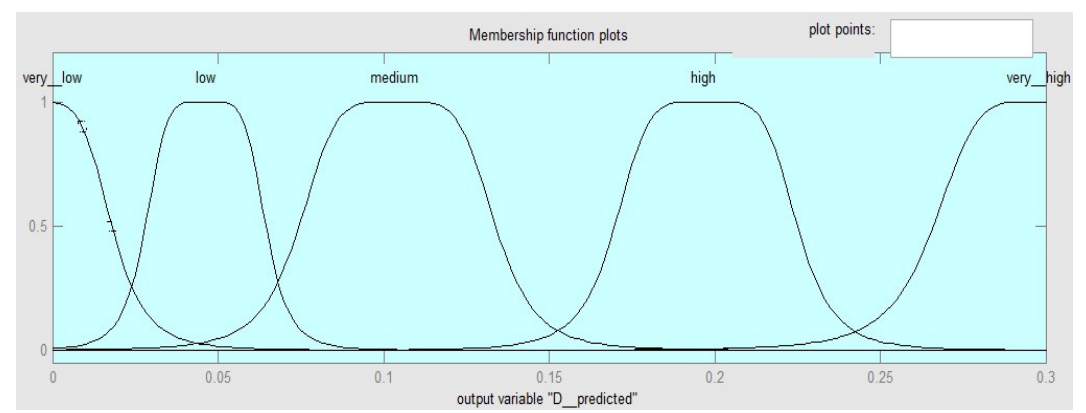

(c)

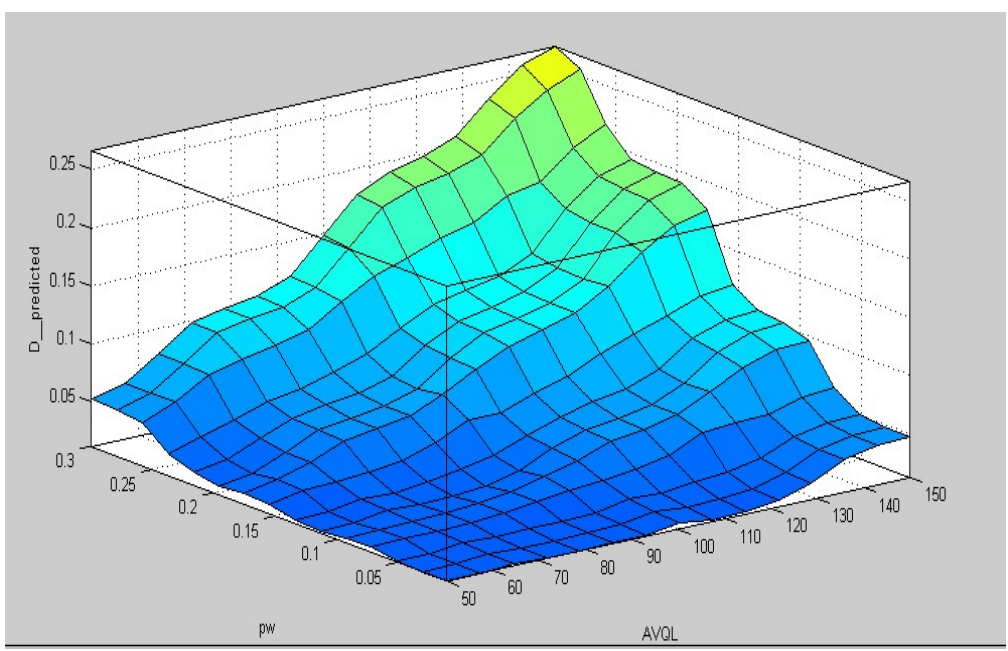

(d)

Figure 4. A Fuzzy-Logic Adaptive Queuing based RED scheme: Mamdani Fuzzy -Logic Based on General Bell function (a) membership of linguistic variable $A V Q L$ (b) membership linguistic variable $P_{w} \quad$ (c) membership of output $D_{p}$, and (D) Fuzzy logic system decision surface

\section{Throughput Performance}

\subsection{Heuristic TCP Formula}

There are several formulas that have been proposed to approximate the behaviour of TCP throughput [3][24][27]. In this framework, in order to estimate the network throughput, we can apply the heuristic TCP formula derived by Zorzi et al. [27] for the most accurate performance in wireless communications. According to it, the heuristic throughput of a single connection flow over Rayleigh fading channel can be defined by

$$
R_{k} \approx R_{s}\left\{\begin{array}{lll}
10^{X_{s} /\left(X_{s}+1\right)} & \text { if } & \xi_{w}>\xi_{s} \\
1 & \text { if } \quad \xi_{w} \leq \xi_{s}
\end{array}\right.
$$

with,

$$
X_{s}=10^{\varepsilon \cdot \ln \left(\frac{1}{\xi_{w}-\xi_{s}}-1\right)}
$$


- $R_{k}$ is the current average throughput at the sample $k$.

- $R_{s}$ is the average throughput for error-free links and it can also denote the available or source bit rate in [bps].

- $\quad \xi_{w}=1+\left[\log _{10}\left(P_{w}\right) / 3\right]$

- $\xi_{s}=1 /\left(\theta . f_{d}+\beta\right)-\mathrm{K}$.

Note that $f_{d}$ denotes the Doppler frequency used to model the Rayleigh fading, whereas $\theta, \beta$ and $\mathrm{K}$ are constants. For numerical values we can use $\theta=1.39, \beta=2.78, \mathrm{~K}=0.03$, and a typical value for $f_{d}$ is $f_{d}=6 \mathrm{~Hz}$. In addition, it is worth noting that the throughput expression given in (4) is a heuristic function obtained by fitting the simulation curves in [27].

To examine the effect of time period of measuring the bit error rate, i.e., the burst errors which accomplishes TCP traffic flow over wireless channel, the smaller value of time period of fading is expected so it can be adapted to the time changing of packet loss rate. However, it must be large enough for the fading speed. The fading speed thus is given by $f_{d}$ (Doppler frequency) as

$$
f_{d}=\frac{v \cdot f_{c}}{c}
$$

where $f_{c}$ is the carrier frequency, $v$ is the vehicle speed $(\mathrm{m} / \mathrm{sec})$, and $c$ is light speed. For 1.9 $\mathrm{GHz}$ in $3 \mathrm{G}$ cellular wireless networks, the above formula yields a Doppler frequency of 6 $\mathrm{Hz} / \mathrm{m} / \mathrm{s}$. Therefore, for the high-speed wireless transmissions (e.g., in the order of Mbps), the fading speed can be viewed as roughly constant value for the time period of measurement of bit error rate. As a consequence, such fading effect is required to be investigated for the measured packet loss rate due to the burst bit errors.

According to our proposed FLAQ-RED controller described in Figure 2, we adopt the TCP layer packet error rate (PER) as the feedback information on the current degree of wireless environment reliability. Once the TCP layer drops a certain number of packets, the receiver (MH) informs the sender (BS) to tune the queue size in terms of $A V Q L$, and consequently finding the predicted $D_{p}$, which is the target packet loss rate required to optimize and maximize the TCP throughput performance at the mobile receiver. Thus Figure 4 explains the process of FLAQ-RED tuning algorithm based on TCP PER measurement via ACK feedback.

\subsection{Buffer Queue Model Based On RTT}

In our FLAQ-RED scheme, firstly we let $B_{k} \leq B_{w}$ where $B_{w}$ stands for available wireless channel bandwidth and $B_{k}$ is the current available bandwidth [24]. Then, the buffer queue length $(Q)$ is expressed at the sampling instant $(k+1)$ by the following equation [28],

$$
Q_{k+1}=Q_{k}+R T T \times\left(R_{k-1}-B_{k}\right)
$$

where $R T T$ denotes the current round-trip time which is also the sampling interval and $R_{k-1}$ is the TCP source rate of BS during the previous RTT. This allows for the round-trip delay in the control loop. In (6), $B_{k}$ denotes the available network bandwidth. Since the heuristic TCP throughput formula defined in (1) does not directly depend on RTT, therefore we are able to assume that the available network bandwidth equals the previous TCP source rate, then the resultant average queue length $(A V Q L)$ in (6) is being $Q_{k+1}=Q_{k}$. 
International Journal of Computer Networks \& Communications (IJCNC) Vol.4, No.3, May 2012

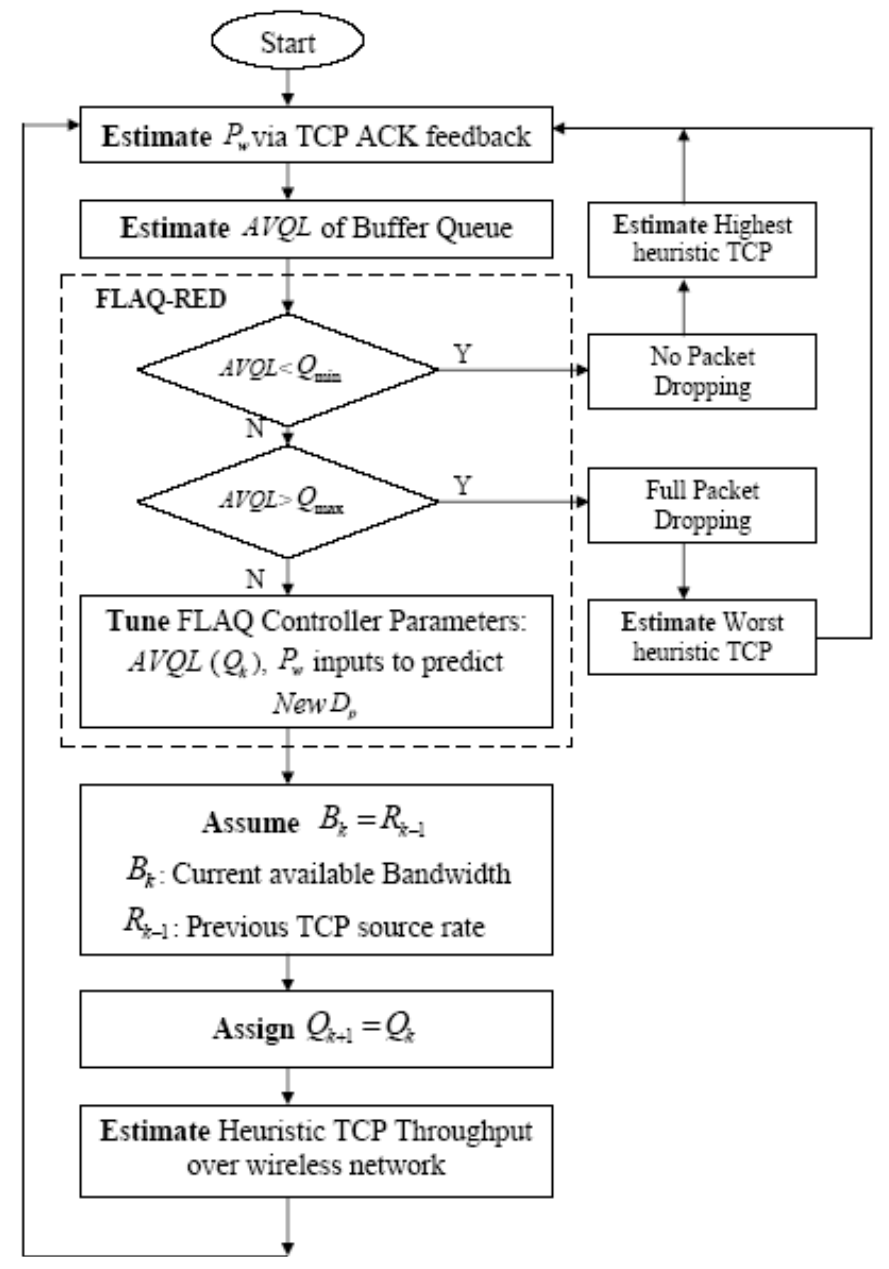

Figure 4. Flow chart of control loop of the proposed FLAQ-RED algorithm to estimate heuristic TCP throughput performance

\section{SimUlation RESUltS}

\subsection{Network Settings}

Using Table 3, we have conducted our simulation in UMTS cellular wireless network in order to investigate the improvement in TCP performance over a wireless link of BS and MH nodes.

Table (3): Simulation Parameters [22][24]

\begin{tabular}{ll}
\hline Parameters & Value \\
\hline$R T T_{\min }$ & $168 \mathrm{~ms}$ \\
$B_{w}$ & $1 \mathrm{Mbps}$ \\
Carrier frequency & $1.9 \mathrm{GHz}$ \\
Source bit rate & $1 \mathrm{Mbps}$ \\
Buffer Queue Size & $200[$ Packet] \\
$Q_{\min }, Q_{\text {max }}$ & $50[$ Packet] 150 [Packet] \\
Mobile speed & $1 \mathrm{~m} / \mathrm{s}-10 \mathrm{~m} / \mathrm{s}$ \\
$P_{w}$ & $5 \%-30 \%$ \\
\hline
\end{tabular}


International Journal of Computer Networks \& Communications (IJCNC) Vol.4, No.3, May 2012

\subsection{Performance Evaluation}

We performed our simulation experiments using the numerical values described in Table 1 as follows. MATLAB mfile and fuzzy logic tool box are used to simulate our proposed FLAQRED scheme. We conducted our experiments to investigate two cases of user's mobility as follows: (1) the stationary receiver (i.e., there is no Doppler effect) and (2) The mobile receiver (i.e., there is Doppler frequency) over time-varying wireless channel.

The first experiment was on predicting the dropping packet rate at the BS router using our proposed FLAQ-RED scheme. The wireless packet loss rate and the measured average queue length are considered as inputs of FLAQ-RED controller. The range of predefined wireless packet rate is changed over the range of 5\% -30\%. Using General Bell fuzzy logic model we were able to estimate the predicted dropping packet rate for the next transmission. In this model, we assume the maximum buffer size is up to 200 packets, and the two queue threshold levels are $Q_{\min }$ and $Q_{\max }$ as defined in Table 1, respectively.

Figure 6 displays the normalized throughput vs. the AVQL for various values of the predefined $P_{w}$ for stationary receiver over wireless channel. It is found that our FLAQ-RED introduces an excellent performance especially for high values of the predefined $P_{w}$. The maximum achievable throughput of 0.9999 can be obtained when $A V Q L$ does not exceed the two queue thresholds and $P_{w}$ equals 5\%. In addition, the higher values of $P_{w}$, say $30 \%$, the resultant throughput will not be less than 0.55 .

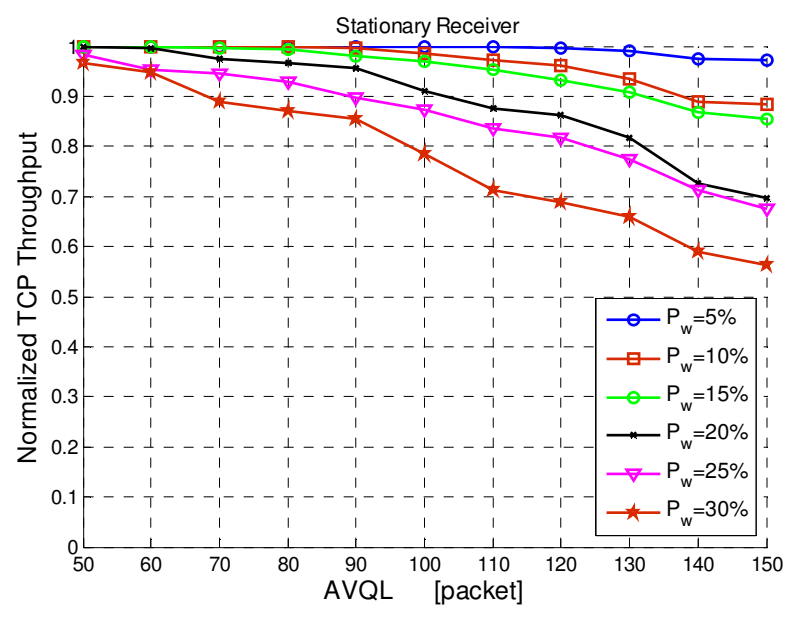

Figure 6. Normalized TCP throughput vs. AVQL for various values of packet loss rate

Table 4 summarizes the performance comparison of our proposed FLAQ-RED scheme. The normalized TCP throughput is evaluated at the predicted packet dropping rate for the two queue length thresholds, $Q_{\min }=50$ and $Q_{\max }=150$, respectively. The results obtained indicate that FLAQ-RED is able to tune its inputs to achieve the maximum throughput at $Q_{\min }$ and the minimum throughput at $Q_{\max }$, respectively. For instance, once $P_{w}$ increases to $30 \%$ the worst achievable heuristic throughput becomes 0.5638 at $Q_{\max }$. Then, using RED algorithm, any increment beyond $Q_{\max }$ takes place; there is an increment in $D_{p}$ and consequently the predicted throughput drops immediately down to be in a worst state over the network. 
International Journal of Computer Networks \& Communications (IJCNC) Vol.4, No.3, May 2012

Figure 7 reveals the effect of user's mobility on the TCP performance for the two predefined $\mathrm{P}_{\mathrm{w}}$ of $5 \%$ and $10 \%$, respectively. The results obtained uses UMTS cellular network assuming the operating frequency of $1.9 \mathrm{GHz}$ and the mobile speed varies up to $10 \mathrm{~m} / \mathrm{s}$. It is clearly shown

Table (4): Heuristic TCP Throughput Performance of FLAQ-RED at Stationary Receiver

\begin{tabular}{|c|c|c|}
\hline \multirow{2}{*}{$\begin{array}{c}\text { Predefined } \\
P_{w}\end{array}$} & \multicolumn{2}{|c|}{ Predicted $D_{P}$, Heuristic TCP Throughput } \\
\cline { 2 - 3 } & $Q_{\min }=50$ & $Q_{\max }=150$ \\
\hline $5 \%$ & $1.29 \%, 0.9999$ & $4.96 \%, 0.9714$ \\
\hline $10 \%$ & $1.40 \%, 0.9998$ & $10.0 \%, 0.8835$ \\
\hline $15 \%$ & $1.74 \%, 0.9992$ & $11.4 \%, 0.8551$ \\
\hline $20 \%$ & $1.91 \%, 0.9987$ & $19.1 \%, 0.6976$ \\
\hline $25 \%$ & $3.97 \%, 0.9835$ & $20.2 \%, 0.6764$ \\
\hline $30 \%$ & $5.24 \%, 0.9675$ & $26.6 \%, 0.5638$ \\
\hline
\end{tabular}

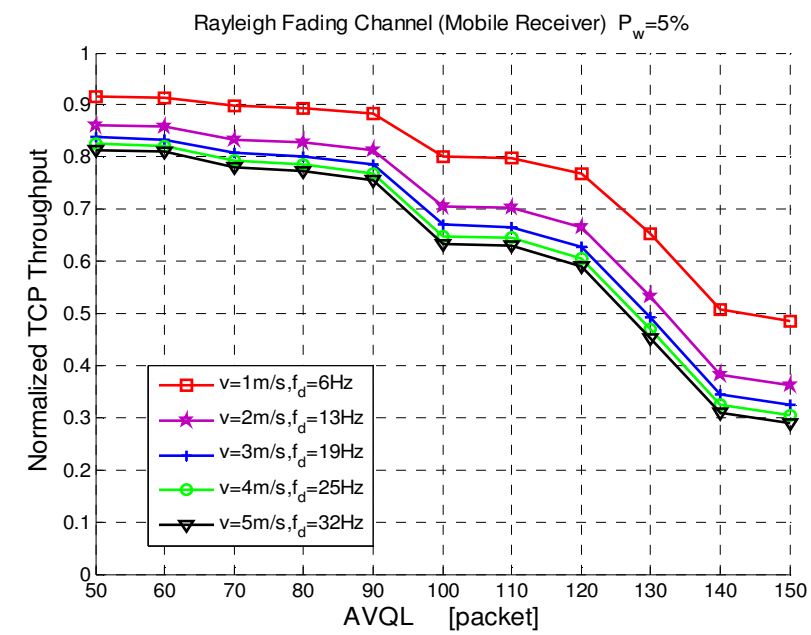

(a)

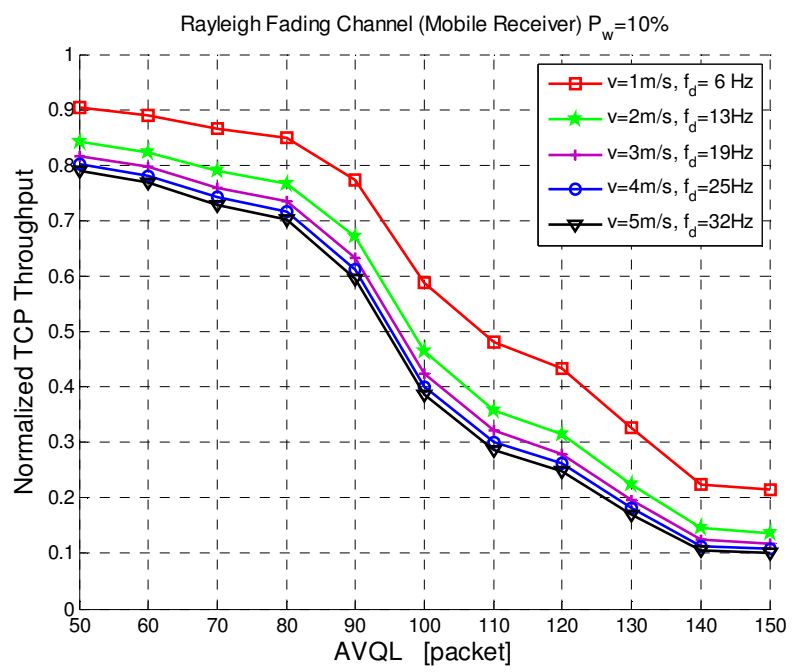

(b)

Figure 7. Normalized TCP throughput vs. AVQL over time-varying fading channel (mobile receiver) (a) $\mathrm{P}_{w}=5 \%$ and (b) $\mathrm{P}_{\mathrm{w}}=10 \%$ 
International Journal of Computer Networks \& Communications (IJCNC) Vol.4, No.3, May 2012

that once the mobile speed increases up to $5 \mathrm{~m} / \mathrm{s}$, the heuristic TCP throughput rapidly degrades especially when AVQL achieves $\mathrm{Q}_{\max }$, where all arriving packets are dropped. Then, the lowest TCP throughput achieves 0.6 and 0.1 , respectively, when $\mathrm{P}_{\mathrm{w}}$ changes from $5 \%$ to $10 \%$ and $A V Q L$ becomes $\mathrm{Q}_{\max }$. Moreover, TCP throughput performance becomes independent (i.e., close to i.i.d case) when Doppler frequency closes to $5 \mathrm{~m} / \mathrm{s}$ or higher. Finally, Tables 5 and 6 both can summarize some details on performance comparison of the proposed FLAQ-RED under different channel conditions.

Table (5): Heuristic TCP throughput performance of FLAQ-RED over wireless Rayleigh fading channel at $P_{w}=5 \%$

\begin{tabular}{|c|c|c|c|}
\hline Mobile & Doppler & \multicolumn{2}{|c|}{ Predicted $D_{P}$, Optimal Heuristic } \\
Speed & Frequency & TCP Throughput & \\
\cline { 3 - 4 }$v(\mathrm{~m} / \mathrm{s})$ & $(\mathrm{Hz})$ & $Q_{\min }=50$ & $Q_{\max }=150$ \\
\hline 1 & 6 & $1.29 \%, 0.9162$ & $4.96 \%, 0.4843$ \\
\hline 2 & 13 & $1.29 \%, 0.8610$ & $4.96 \%, 0.3620$ \\
\hline 3 & 19 & $1.29 \%, 0.8383$ & $4.96 \%, 0.3246$ \\
\hline 4 & 25 & $1.29 \%, 0.8246$ & $4.96 \%, 0.3046$ \\
\hline
\end{tabular}

Table (6): Heuristic TCP throughput performance of FLAQ-RED over wireless Rayleigh fading channel at $P_{w}=10 \%$

\begin{tabular}{|c|c|c|c|}
\hline \multirow{2}{*}{$\begin{array}{l}\text { Mobile } \\
\text { Speed } \\
v(\mathrm{~m} / \mathrm{s})\end{array}$} & \multirow{2}{*}{$\begin{array}{l}\text { Doppler } \\
\text { Frequency } \\
(H z)\end{array}$} & \multicolumn{2}{|c|}{ Predicted $D_{P}$, Optimal Heuristic TCP Throughput } \\
\hline & & $Q_{\min }=50$ & $Q_{\max }=150$ \\
\hline 1 & 6 & $1.40 \%, 0.9027$ & $10.0 \%, 0.2145$ \\
\hline 2 & 13 & $1.40 \%, 0.8415$ & $10.0 \%, 0.1375$ \\
\hline 3 & 19 & $1.40 \%, 0.8166$ & $10.0 \%, 0.1174$ \\
\hline 4 & 25 & $1.40 \%, 0.8017$ & $10.0 \%, 0.1072$ \\
\hline
\end{tabular}

\section{Conclusions And Future Work}

In this paper, we have proposed an effective scheme called FLAQ-RED to improve the heuristic TCP performance based on active queue management (AQM) in mobile environment. The classical RED algorithm is considered to tune the queue length at the base station node when a predefined packet loss rate is fed via TCP ACK feedback from the mobile host. Fuzzy-logic controller is used to predict the dropping packet rate for the next transmission. The obtained results introduce a good improvement in the link reliability observed by a heuristic TCP throughput over Rayleigh fading channel under different conditions of user's mobility. For further work, this proposed scheme can be extended to investigate the congestion effect of multiple-TCP connection flows. Furthermore, the effect of dynamic packet sizes on this FLAQRED scheme can be taken into account in order to improve the multimedia traffic over wireless environment. The scheme can also be improved by applying the weighted RED to provide different multiple-queue threshold levels for traffic classes.

\section{REFERENCES}

[1] Welzl M. "Network Congestion Control: Managing Internet Traffic," 282 pages, July 2005.

[2] J. Widmer, C. Boutoemans, J. Y. Boudec, (2004), "End-to-End Congestion Control for TCPFriendly Flows with Variable Packet Size”, ACM SIGCOMM 2004. 
International Journal of Computer Networks \& Communications (IJCNC) Vol.4, No.3, May 2012

[3] K. Zhou, K. L., Yeung, and V. O.K. Li, (2006), "Nonlinear RED: A Simple Yet Efficient Active Queue Management Scheme," Computer Networks, vol. 50, pp. 3784-3794.

[4] E. Dahiman, S. Parkvall, J. Skold, P. Beming,(2008), 3G Evolution: HSPA and LTE for Mobile Broadband, $2^{\text {nd }}$ ed., Academic Press.

[5] P. Dalal, N. Kothari and K.S. Dasgupta, (2011), "Improving TCP Performance over Wireless Network with Frequent Disconnections," International J. of Computer Networks \& Communications (IJCNC), vol. 3, no.6, pp. 169-184.

[6] A. Akella, S. Seshan, and A. Shaikh, (2003), “An Empirical Evaluation of Wide-Area Internet Bottlenecks," Proc. of ACM Internet Measurement Conf. (IMC).

[7] M. Hirabaru, "Impact of Bottleneck Queue Size on TCP Protocols and Its Measurement," (2006) IEICE Trans. on Information and Systems, vol E89-D, no.1, pp. 132-138.

[8] J. Liu and M. Crovella, (2001), "Using Loss Pairs to Discover Network Properties," ACM SIGCOMM Workshop on Internet Measurement, pp. 127-138.

[9] S. C. F. Chan and J. Y. B. Lee, (2011), "A Novel Link Buffer Size Estimation Algorithm for Bandwidth Varying Mobile Data Networks," Proc. of IEEE $7^{\text {th }}$ Inter. Conf. on Wireless and Mobile Computing, Networking and Communications ( WiMob).

[10] C. P. Fu and S. C. Liew, (2003), "TCP Veno: TCP Enhancement for Wireless Access Networks," IEEE J. of Selected Areas in Communications, vol.21, no.2.

[11] H. Abdel-Jaber, M. Mahafzah, F. Thabtah, and M. Woodward, (2008), "Fuzzy Logic Controller of Random Early Detection based on Average Queue Length and Packet Loss Rate", Proc. of SPECTS 2008.

[12] M .H. Y. Moghaddam, (2010), “A Fuzzy Active Queue Management Mechanism for Internet Congestion Control," Third International Workshop on Advanced Computational Intelligence, pp.203- 208, August.

[13] C. Chrysostomou, A. Pitsillides, L. Rossides, M. Polycarpou, and A. Sekercioglu, (2003), "Fuzzy Logic Controlled RED: Congestion Control in TCP/IP Differential Services Networks", Soft Computing, Springer.

[14] T. B. Reddy, A. Ahammed, and R. Nanu, (2009), "Performance Comparison of Active Queue Management Techniques," Inter. J. of Computer Science and Networks Security (IJCNS), vol. 9, no.2.

[15] J. D. Mallapur, S. S. Mavi, and D. H. Rao, (2010), "Fuzzy-Based Approach for Packet Dropping in Wireless Networks," Int. J. of Advanced Networking and Applications, vol. 1, no. 5, pp. 301306.

[16] S. Floyd and V. Jacobson, (1993), "Random Early Detection Gateways for Congestion Avoidance", IEEE/ACM Transactions on Networking, 1(4):397- 413.

[17] T. Vu, (2003), "Effect of RED and Different Packet sizes on Multimedia Performance over Wireless Networks," TU llmenau, Germany.

[18] A. E. Abharian and M. Alireza, (2011), "Hybrid GA-BF Based Intelligent PID Active Queue Management Control Design for TCP Network," IEEE Inter. Conference.

[19] A. Athuraliya, S. H. Low, V.H. Li, et al., (2001), "REM: Active Queue Management," IEEE Network, vol. 15, pp. 48-53.

[20] T. Shikama, (2010), "Mitigation of Bursty Packets By a TCP Proxy Improving TCP Performance in a Wired and Wireless Network," IEEE Globcom Workshop On Complex and Communication Networks.

[21] M. Miyoshi M. Sugano, and M Murata, (2002), "Improving TCP Performance for Wireless Cellular Networks by Adaptive FEC Combined with Explicit Loss Notification," IEICE Trans. Comm. VOL-E85, no. 10, pp.2208-2213. 
International Journal of Computer Networks \& Communications (IJCNC) Vol.4, No.3, May 2012

[22] Q. Liu, S. Zhou, and G. B. Giannahis, (2005), "Queuing with Adaptive Modulation and Coding over Wireless Links: Cross-Layer Analysis and Design," IEEE Trans. Wireless Comm., vol. 4, no. 3 .

[23] H. Han (2011), "Performance Improvement of TCP Reno Based on Monitoring the Wireless Packet Loss Rate" Physics \& Electronics Engineering Department, Xiangfan University, China.

[24] M. Chen and A. Zakhor, (2005), "Rate Control for Streaming Video over Wireless", IEEE Wireless Communication, pp. 32-41.

[25] J. Jantzen, “Design of Fuzzy Controllers.” ww.iau.dtu.dk/ jij/pubs/design.pdf.

[26] K. Michels, F. Klawonn, R. Kruse, A. Nürnberger, (2006), "Fuzzy Control: Fundamentals, Stability and Design of Fuzzy Controllers", Springer-Verlag Berlin Heidelberg.

[27] M. Zorzi, M. Rossi, and G. Mazzini, (2002), "Throughput and energy performance of TCP on a wideband CDMA air interface," Wireless Communications and Mobile Computing, vol. 2, no. 1. pp. 71-84.

[28] H. Sirisena, M. Hassan, and A. Haider, (2002), “Optimal TCP Congestion Control,” Proc. ICT'02, pp. 732-736.

\section{Authors Short Biography}

Ghaida A. AL-Suhail is an Assistant Professor at the University of Basrah, Computer Engineering Department, Basrah, IRAQ. She received the PhD degree in Electrical Engineering from the University of Basrah, in 2007. Her current research interests include wireless networks and communications, mobile multimedia, image processing, Internet Protocols, Cross-layer design, Quality of Service, modelling and Simulation, and mobile computing. She has been a Fulbright Scholar 2011 at the Michigan State University (MSU) in USA and Endeavour Fellowship 2009 Scholar at the Australian National University (ANU), RSISE, in Australia. She published several papers in International Journals and Conferences like JMM, IJMCMC, WSEAS'06, ICARCV'06, MoMM08/2009, ICSPCS7/8/9/2010 (Australia and USA), WiMo2011 (Turkey) and EPC-IQ01 and CCIT2012 (IRAQ).

Turki Y. Abdalla received the Ph.D. degree in Electrical Engineering from the University of Basrah, Iraq, in 1999. He has been a professor at the department of computer engineering in the University of Basrah since 2002. His current research interests include learning control, robust control, Fuzzy control, Neural Network, wavelet Network, Genetic algorithm , Particle swarm optimization, robot planning, Multiple robot system, and Wireless sensor Network, He is an author and co- author of more than 40 journal and conference papers. He has supervised several MSc, and $\mathrm{PhD}$ thesis in computer and electrical Engineering.

Omar Majid received B.E. Degree (2010) in Computer Engineering Department. He is currently MSc. Student at the Computer Engineering Department, University of Basrah, IRAQ. His research interests include network performance evaluation, TCP protocol, cellular networks and fuzzy logic systems.
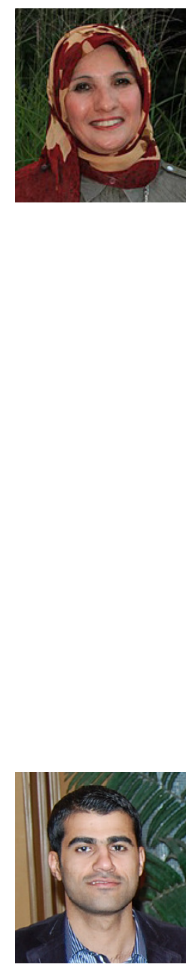